\title{
Fabrication of silicon nanostructures for application in photonics
}

(C) A.N. Kamalieva ${ }^{1}$, N.A. Toropov ${ }^{1}$, T.A. Vartanyan ${ }^{1, \pi}$, M.A. Baranov ${ }^{1}$, P.S. Parfenov ${ }^{1}$, K.V. Bogdanov ${ }^{1}$, Y.A. Zharova ${ }^{1,2}$, V.A. Tolmachev ${ }^{2}$

${ }^{1}$ ITMO University,

197101 St. Petersburg, Russia

${ }^{2}$ loffe Institute,

194021 St. Petersburg, Russia

ฯ E-mail: Tigran.Vartanyan@mail.ru

Silicon is the primary material of modern electronics. It also possesses bright potentials for applications in nanophotonics. At the same time optical properties of bulk silicon do not fully satisfy requirements imposed on them. Fortunately, properties of silicon nanostructures strongly depend on their shapes and sizes. In this regard, of special interest is the development of fabrication and post-processing methods of silicon nanostructures. In this contribution we propose a method for silicon nanostructures fabrication combining the technique of high-vacuum deposition with metal-assisted chemical etching. SEM images as well as ellipsometry, Raman scattering and optical spectroscopy data prove that the desired structural changes were obtained.

\section{Acknowledgement}

Authors thank the next foundations for financial support: the RFBR (16-02-00932 A, and 17-02-01116 A), the Government of Russia (074-U01), Presidential Grant (MK-228.2017.2), and Ministry of education and science of Russian Federation (3.4903.2017/6.7). 